\title{
Steady Stagnation Point Flow and Heat Transfer Over a Shrinking Sheet with Induced Magnetic Field
}

\author{
A. Sinha \\ Department of Mathematics Jadavpur University, Jadavpur-700032, India \\ Email: aniruddha.sinha07@gmail.com
}

(Received November 18, 2013; accepted December 18, 2013)

\begin{abstract}
The problem of the steady magnetohydrodynamic (MHD) stagnation-point flow of an incompressible, viscous and electrically conducting fluid over a shrinking sheet is studied. The effects of an induced magnetic field and thermal radiation are taken into account. Velocity and thermal slip conditions have also been incorporated in the study. The nonlinear partial differential equations are transformed into ordinary differential equations via the similarity transformation. The transformed boundary layer equations are solved numerically using Newton's linearization method. Computational results for the variation in velocity, temperature, skin-friction co-efficient and Nusselt number are presented graphically and in tabular form. Study reveals that the surface velocity gradient and heat transfer are enhanced by decreasing magnetic parameter.
\end{abstract}

Keywords: Shrinking sheet; Stagnation flow, Induced magnetic field; Slip effects; Boundary layer.

\section{INTRODUCTION}

A class of flow problems with obvious relevance to numerous applications in industrial manufacturing processes is the flow induced by the stretching motion of a flat elastic sheet. Such flow situations are encountered, for example, in aerodynamic extrusion of plastic and rubber sheets, melt-spinning, hot rolling, wire drawing, glass-fiber production, polymer sheets, cooling of a large metallic plate in a bath which may be an electrolyte, etc. During its manufacturing process, a stretched sheet interacts with the ambient fluid both thermally and mechanically. The study of heat transfer and flow field is necessary for determining the quality of the final products of such processes as explained by Karwe and Jaluria (1988, 1991). Crane (1970) was the first who studied the steady two-dimensional incompressible boundary layer flow of a Newtonian fluid caused by the stretching of an elastic flat sheet which moves in its own plane with a velocity varying linearly with the distance from a fixed point due to the application of a uniform stress. This problem is particularly interesting since an exact closed form solution of the two-dimensional Navier-Stokes equations has been obtained.

In recent years, some interest has been given to investigate the flow over a shrinking sheet, where the sheet is stretched toward a slot and it would cause a velocity away from the sheet. However, in certain situations, the shrinking sheet solutions do not exist since the vorticity can not be confined in a boundary layer. A pioneering paper on this problem has been published by Miklavcic and Wang (2006). From the physical grounds vorticity (rotation or non-potential) flow over the shrinking sheet is not confined within a boundary layer, and the flow is unlikely to exist unless adequate suction on the boundary is imposed (Miklavcic and Wang (2006)). Fang and Zhang (2009) obtained an analytical solution for the thermal boundary layers with suction over shrinking sheet. The shrinking sheet problem has also been extended to micropolar fluid (Ishak et al. (2008)) as well as magnetohydrodynamic fluid (Sajid et al. (2008)).

During the past century,many engineering problems of fluid mechanics have been solved by using the boundary-layer theory and the results compare well with the experimental observations for Newtonian fluids (Schlichting and Gersten (2000)). An extension of the boundary layer theory to non-Newtonian fluids is found to be rather difficult (Rajagopal et al. (1980)). This difficulty is caused by the diversity of non-Newtonian fluids in their constitutive behaviour and simultaneous viscous and elastic properties. Consequently, most studies on non-Newtonian boundary layers have used simple rheological models such that these two effects can be taken into account separately. Recently, several researchers (Singh et al. (2012), Sharma and Singh (2009), Rashidi and Erfani (2010), Erfani et al. (2011)) analytically as well as numerically studied the boundary layer flow in the stagnation point region of a two dimensional body. Hiemenz (1911) was the first to study the two-dimensional (2D) 
stagnation-point flow against an infinite flat plate. $\mathrm{He}$ found an exact solution to the governing Navier-Stokes equations. Mahapatra and Gupta (2004) considered the boundary layer flow near the stagnation-point on a stretching sheet, where time dependence is also taken into account. Recently, Zhu et al. (2009) presented the analytical solutions of stagnation-point flow over a stretching sheet. These are few examples for the problem where the induced magnetic field is negligible.

To date, very little work has been done on the boundary layer flow and heat transfer with the consideration of the induced magnetic field. Kumari et al. (2009) investigated the MHD flow and heat transfer over a stretching surface by considering the effect of the induced magnetic field. Rashidi et al. (2011) investigated the simultaneous effects of partial slip and thermal-diffusion and diffusion-thermo on MHD flow due to a rotating disk.

Owing to the above mentioned studies, in this paper, we have investigated a numerical solution on a MHD stagnation-point flow with heat transfer over a shrinking sheet in the presence of magnetic field having interest to the engineering community and to the investigators dealing with the problem in geophysics, astrophysics, electrochemistry and polymer processing. The effects of an induced magnetic field and thermal radiation are taken into account. In this paper, we have investigated the effects of slip velocity and thermal slip on an electrically conducting viscous incompressible fluid. Boundary layer theory is applied to simplify the equations that govern the fluid motion, induced magnetic field and energy. Computational results presented through graphs put forward theoretical estimates of the influence of various parameters involved in the study. The results reported here are applicable to a variety of bioengineering problems.

\section{MATHEMATICAL ANALYSIS}

Let us Consider a steady, two-dimensional flow and heat transfer of an incompressible electrically conducting fluid near the stagnation point on a heated shrinking sheet in the presence of a free stream $u_{e}(x)$ and uniform ambient temperature $T_{\infty}$. The x-axis runs along the shrinking surface in the direction of motion and the $y$-axis is perpendicular to it, as shown in Fig. 1. The wall shrinking sheet velocity $u_{w}(x)$ and the wall temperature $T_{w}(x)$ are are proportional to the distance (x) from the stagnation point, where $u_{e}(x)=a x$ and $T_{w}(x)=T_{\infty}+T_{0}(x / l)$,

where $a$ is a constant, $T_{0}$ is the reference temperature. It is also assumed that an induced magnetic field of strength $H_{0}$ (say) acts in the direction normal to the surface. It is supposed that at the wall, the normal component of the induced magnetic field $\mathrm{H}_{2}$ has a vanishing value, while the parallel component $H_{1}$ assumes the value $H_{0}$.

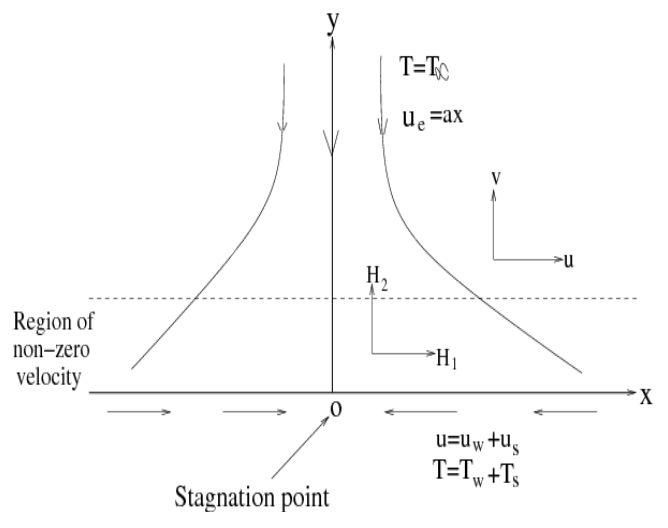

Fig. 1. Physical sketch of the problem

According to the boundary layer approximations, the basic equations of the problem can be written as follows (Datti et al. (2004), Sharma and Singh (2009), Singh et al. (2012))

$$
\begin{aligned}
& \frac{\partial u}{\partial x}+\frac{\partial v}{\partial y}=0 \\
& \frac{\partial H_{1}}{\partial x}+\frac{\partial H_{2}}{\partial y}=0 \\
& u \frac{\partial u}{\partial x}+v \frac{\partial u}{\partial y}-\frac{\mu_{e}}{4 \pi \rho}\left(H_{1} \frac{\partial H_{1}}{\partial x}+H_{2} \frac{\partial H_{1}}{\partial y}\right)=\left(u_{e} \frac{d u_{e}}{d x}-\right. \\
& \left.\frac{\mu_{e} H_{e}}{4 \pi \rho} \frac{d H_{e}}{d x}\right)+v \frac{\partial^{2} u}{\partial y^{2}} \\
& u \frac{\partial H_{1}}{\partial x}+v \frac{\partial H_{1}}{\partial y}-H_{1} \frac{\partial u}{\partial x}-H_{2} \frac{\partial u}{\partial y}=\eta_{0} \frac{\partial^{2} H_{1}}{\partial y^{2}} \\
& u \frac{\partial T}{\partial x}+v \frac{\partial T}{\partial y}=\frac{k}{\rho c} \frac{\partial^{2} T}{\partial y^{2}}-\frac{1}{\rho c} \frac{\partial q_{r}}{\partial y}
\end{aligned}
$$

Where $\mu$ is the coefficient of viscosity, $\rho$ is the fluid density, $v$ being the kinematic coefficient of viscosity, $\eta_{0}$ represents magnetic diffusivity, $k$ being the thermal conductivity, $c_{p}$ is the specific heat at constant pressure and $\mu_{e}$ is the Magnetic permeability.

The boundary conditions for the present problem can be written as

$$
\begin{aligned}
& u=u_{w}+u_{s}, \quad v=0, \quad \frac{\partial H_{1}}{\partial y}=H_{2}=0, \\
& T=T_{w}+T_{s} \quad \text { at } \quad y=0
\end{aligned}
$$

$u=u_{e}(x)=a x, H_{1}=H_{e}(x)=H_{0}(x / l)$,

$T=T_{\infty} \quad$ at $y \rightarrow \infty$

in which $u_{w}=-c x \quad, \quad u_{s}=\mu N \frac{\partial u}{\partial y}$, $T_{w}=T_{\infty}+T_{0}(x / l)$ and $T_{S}=K \frac{\partial T}{\partial y}$,

$a$ an $c$ being positive constants. $N$ is the velocity slip factor and $K$ is the thermal slip factor.

The radiative heat flux term by using the Rosseland 
A. Sinha / JAFM, Vol. 7, No. 4, pp. 703-710, 2014.

approximation (Singh et al. (2012)) is given by

$q_{r}=-\frac{4 \sigma^{*}}{3 k^{*}} \frac{\partial T^{4}}{\partial y}$

where $\sigma^{*}$ is the Stefan-Boltzmann constant and $k *$ being the mean absorption coefficient.

Assuming that the differences in temperature within the flow are such that $T^{4}$ can be expressed as a linear combination of the temperature, we expand $T^{4}$ in Taylor's series about $T_{\infty}$ as follows

$T^{4}=T_{\infty}^{4}+4 T_{\infty}^{3}\left(T-T_{\infty}\right)+6 T_{\infty}^{2}\left(T-T_{\infty}\right)^{2}+\cdots$

and neglecting higher order terms beyond the first degree in $\left(T-T_{\infty}\right)$, we get

$T^{4}=4 T_{\infty}^{3} T-3 T_{\infty}^{4}$

Let us introduce the following dimensionless variables:

$\psi=(c v)^{1 / 2} x f(\eta), \quad \theta=\frac{T-T_{\infty}}{T_{w}-T_{\infty}}, \quad \eta=\left(\frac{c}{v}\right)^{1 / 2} y$

$H_{1}=H_{0}(x / l) g^{\prime}(\eta), \quad H_{2}=-\left(\frac{v}{l^{2} c}\right)^{1 / 2} H_{0} g(\eta)$

The velocity components $u$ and $v$ can be

obtained from the stream function $\psi$ as $u=\frac{\partial \psi}{\partial y}$

and $v=-\frac{\partial \psi}{\partial x}$.

It may be noted that the continuity Eq.(1) is automatically satisfied.

Then substituting Eqs. (8) to (10) into Eqs. (3) to (5) yields the following dimensionless equations:

$f^{\prime \prime \prime}+f f^{\prime \prime}-f^{\prime 2}+\frac{a^{2}}{c^{2}}+\beta\left(g^{\prime 2}-g g^{\prime \prime}-1\right)=0$

$\lambda g^{\prime \prime \prime}+f g^{\prime \prime}-f^{\prime \prime} g=0$

and

$$
\frac{(1+N r)}{\operatorname{Pr}} \theta^{\prime \prime}+f \theta^{\prime}=0
$$

In the above equations, primes denote differentiation with respect to $\eta$. The non-dimensional parameters appeared in Eqs (11) to (13) are defined as $\beta=\frac{\mu_{e}}{4 \pi \rho}\left(\frac{H_{0}}{l c}\right)^{2}$ is the magnetic parameter, $\lambda=\frac{\eta_{0}}{v}$ is the reciprocal magnetic Prandtl number, $N r=\frac{16 \sigma^{\mathrm{a}} T_{\infty}^{3}}{3 k^{\mathrm{a}} k}$ is radiation parameter and $\operatorname{Pr}=\frac{\mu c p}{k}$ is Prandtl number.

The boundary conditions (6) and (7) give rise to

$$
\begin{aligned}
& f(0)=0, \quad f^{\prime}(0)=-1+S_{f} f^{\prime \prime}(0), \quad g(0)= \\
& g^{\prime \prime}(0)=0, \quad \theta(0)=1+S_{t} \theta^{\prime}(0) \\
& f^{\prime}(\infty)=\frac{a}{c}, \quad g^{\prime}(\infty)=1, \\
& \theta(\infty)=0
\end{aligned}
$$

The non-dimensional velocity slip factor $S_{f}$ and non-dimensional thermal slip factor $S_{t}$ are given by $S_{f}=N \rho \sqrt{c v}$ and $S_{t}=K \sqrt{\frac{c}{v}}$.

The magnetic parameter $\beta$, which gives the order of the ratio of the magnetic energy and the kinetic energy per unit volume, is related to the Hartman number $\mathrm{Ha}$ (Hartmann (1937)) and the flow Reynolds number $R e$ and the magnetic Reynolds number $R e_{m}$ as

$$
\begin{aligned}
& \beta=\frac{H a^{2}}{R e R e_{m}}, H a=\mu H_{0} l\left(\frac{\sigma}{\rho}\right)^{1 / 2}, R e= \\
& \frac{(c l) l}{v} \text { and } R e_{m}=4 \pi U_{\infty} l \mu \sigma=\frac{(c l) l}{\mu_{e}}
\end{aligned}
$$

where $l$ is the characteristic length of the shrinking surface comparable with the dimensions of the field.

\section{NUMERICAL PROCEDURE}

Several authors such as Andersson et al. (1992) and Afify (2004) used numerical techniques for the solution of two-point boundary value problems in terms of the Runge-Kutta integration scheme along with the shooting method. Although this method provides satisfactory results, it may fail when applied to problems in which the differential equations are very sensitive to the choice of its missing initial conditions. Moreover, difficulty arises in the case in which one end of the range of integration is at infinity. The end point of integration is usually approximated by replacing a finite representation to this point and it is obtained by estimating a value at which the solution will reach its asymptotic state. On the contrary to the above mentioned numerical method, we used in the present paper that has better stability, simple, accurate and more efficient. The essential features of this technique is that it is based on a finite difference scheme with central differencing and based on the iterative procedure.

We substitute $f^{\prime}=F$ and $g^{\prime}=G$ in (11) and (12), we get

$$
\begin{aligned}
& F^{\prime \prime}+f F^{\prime}-F^{2}+\frac{a^{2}}{c^{2}}+\beta\left(G^{2}-g G^{\prime}-1\right)=0 \\
& \text { and } \\
& \lambda G^{\prime \prime}+f G^{\prime}-F^{\prime} g=0
\end{aligned}
$$

while the boundary conditions (14) and (15) assume the form 


$$
\begin{aligned}
& f(0)=0, \quad F(0)=-1+S_{f} F^{\prime}(0), \quad g(0)=G^{\prime}(0)=0, \\
& F(\infty)=\frac{a}{c}, \quad G(\infty)=1
\end{aligned}
$$

Now using the central difference scheme for derivatives with respect to $\eta$, we write

$\left(P^{\prime}\right)_{i}=\frac{P_{i+1}-P_{i-1}}{2 \delta \eta}+O\left((\delta \eta)^{2}\right)$

and

$$
\left(P^{\prime \prime}\right)_{i}=\frac{P_{i+1}-2 P_{i}+P_{i-1}}{(\delta \eta)^{2}}+O\left((\delta \eta)^{2}\right),
$$

where $P$ stands for $F, G$ and $\theta$; i is the grid index in $\eta$-direction with $\eta_{i}=i * \delta \eta$; $\mathrm{i}=0,1, \ldots . . \mathrm{m}$ and $\delta \eta$ is the increment along the $\eta$ -axis. Newton's linearization method can then be applied to linearize the discretized equations as follows.

When the values of the dependent variables at the $n$ th iteration are known, the corresponding values of these variables at the next iteration can be obtained by using the equation

$$
P_{i}^{n+1}=P_{i}^{n}+\left(\Delta P_{i}\right)^{n},
$$

in which $\left(\Delta P_{i}\right)^{n}$ represents the error at the $n$th iteration, $i=0,1,2, \ldots \ldots, n$. It is worthwhile to mention here that the error $\left(\Delta P_{i}\right)^{n}$ at the boundary is zero, because the values of $P_{i}$ at the boundary are known. Using (22) in (17) and dropping the quadratic terms in $\left(\Delta P_{i}\right)^{n}$, we get a system of block tri-diagonal equations. To solve this tri-diagonal system of equations, we have used the "Tri-diagonal matrix algorithm", usually referred as "Thomas algorithm". It may be mentioned here that instead of this, one could use Gauss elimination method. But in that case, the number of operations would be $\mathrm{m}^{3}$, while in the method that we have employed here, the number of operations is $\mathrm{m}$, where $\mathrm{m}$ is the number of unknowns. Thus the error committed in our method is much less than that in the method of Gauss elimination. In the process of determination of the distribution of the function $f(\eta)$, the accuracy can be defined as the difference between the calculated values of $f(\eta)$ at two successive operations, say $(n+1)$ th and $n$ th. In the present case, the error is equal to

$$
\varepsilon=\left|f^{n+1}(\eta)-f^{n}(\eta)\right|
$$

and is estimated to be less than $10^{-6}$.

\section{NUMERICAL ESTIMATES AND DISCUSSION}

The system of ordinary differential Eqs (11) to (13) subject to the boundary conditions (14) and (15) are solved numerically by employing a finite difference scheme with Newton's linearization method described in the previous section. In order to achieve the numerical solution, it is necessary to assign following values of the dimensionless parameters involved in the present problem under consideration.

$\frac{a}{c}=0.5,0.8,1.5,2.0,2.5$;

$\beta=0.0,0.5,2.0,2.5,4.0,8.0$;

$\lambda=1.0,5.0,10.0,20.0$;

$S_{f}=0.0,0.25,0.5,1.0,2.0$;

$S_{t}=0.0,0.5,1.0,1.5$

$\operatorname{Pr}=0.3,0.5,0.72,1.0,1.5$;

$\mathrm{Nr}=0.0,1.0,2.0,3.0,4.0$.

Numerical computation has been carried out by taking $\delta \eta=0.0125$ with 401 gird points. The results computed have been presented graphically in Figs. 2 to 14. In order to perform grid independent test for the choice of 321 grid points, we repeated the computational procedure by considering a number of mesh sizes by altering the values of $\delta \eta$. If we take $\delta \eta=0.008$ (mess size $=501$ ) or $\delta \eta=0.01$ (mess size $=401$ ) then the figures will be unaltered. It has also been ascertained that for any mesh size less than 321 , the results are inaccurate.

Figures 2 to 4 respectively display the effects of magnetic parameter on axial velocity, induced magnetic field and temperature profiles when $\lambda=1.0, \quad \operatorname{Pr}=0.72, \quad N r=2.0, \quad \frac{a}{c}=2.5$, $S_{f}=0.25$ and $S_{t}=1.0$. Fig. 2 reveals that axial velocity $\left(f^{\prime}\right)$ decreases when the magnetic parameter $\beta$ increases. This is due to the fact that an increase in the value of $\beta$ signifies the increase of Lorentz force and thereby magnitude of velocity reduces. It is also seen from this figure that for large value of magnetic parameter, axial velocity vanishes to a certain point. This point is called point of inflexion. From Fig. 3, it is seen that the induced magnetic field strength decreases as $\beta$ increases. It may be noted from this figure that for any value of $\beta$, induced magnetic field strength increases with the height of the channel. It is noticed from Fig.4 that the magnetic parameter bears the potential to increase the temperature $\theta$ in the boundary layer. This is because of the fact that the induced magnetic field to an electrically conducting fluid gives rise to a resistive type of force known as Lorentz force. This force has the tendency to increase the fluid temperature.

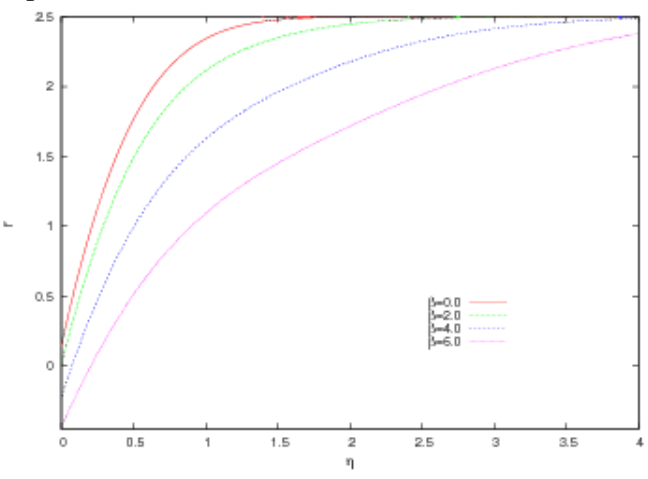


Fig. 2. Axial velocity distribution for different values of $\beta$

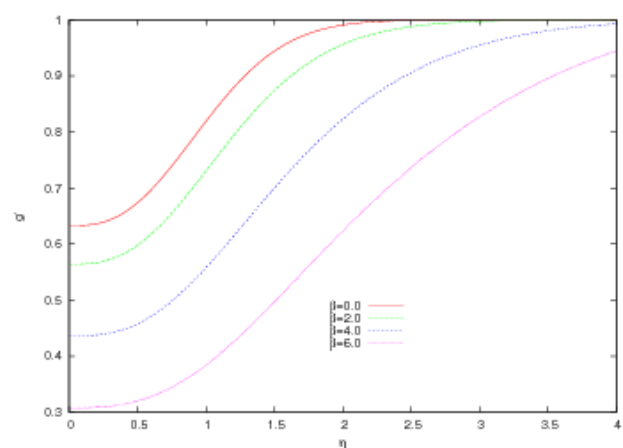

Fig. 3. Variation of induced magnetic field in $x$ -direction for different values of $\beta$

The variation of axial velocity, induced magnetic field and temperature profiles for different values of the ratio $a / c$ is shown in the Figs. 5 to 7 respectively.

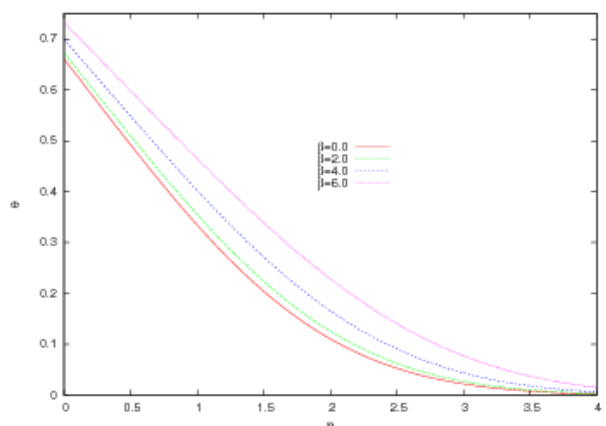

Fig. 4. Temperature distribution for different values of $\beta$

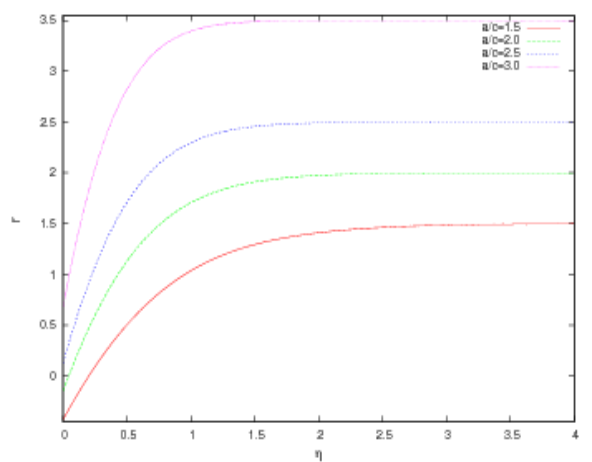

Fig. 5. Axial velocity distribution for different values of $a / c$

It can be seen from Fig. 5 that the boundary layer thickness decreases as $a / c$ increases when we consider $a / c>1$. Physically this phenomenon can be explained as follows: for fixed value of $c$ corresponding to the shrinking of the surface, the increase in $a$ in relation to c implies the increase in the straining motion near the stagnation region that can increase the acceleration of the external stream. Therefore, an increase in $a / c$ has the effect of thinning the boundary layer. From Fig. 6 , it can be seen that all the $g^{\prime}$ profiles increase with the increase in $a / c$. Fig. 7 shows that the the temperature profile decreases in the boundary layer with increasing value of $a / c$. This is because of the fact that the thermal boundary layer decreases with increase in straining motion near the stagnation region.

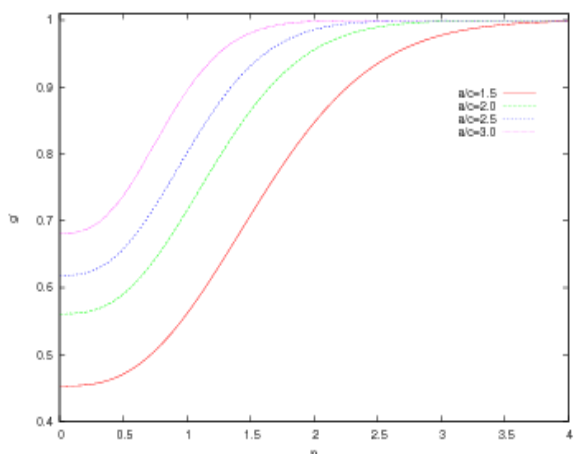

Fig. 6. Variation of induced magnetic field in $x$ -direction for different values of $a / c$

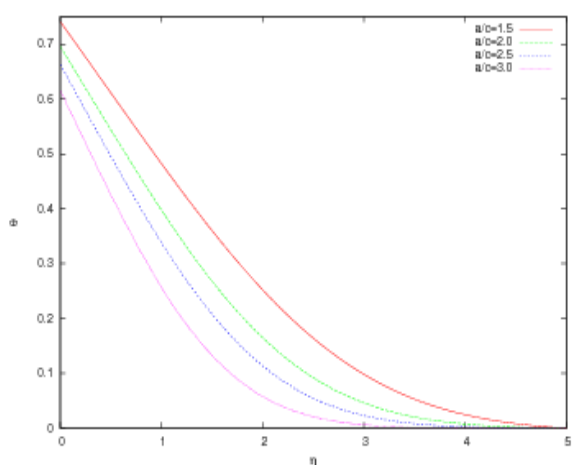

Fig. 7. Temperature distribution for different values of $a / c$

Figures. 8 and 9 illustrate the effects of the velocity slip factor on the velocity and the induced magnetic field profiles when $a / c=2.5$ and $\beta=0.5$. Fig. 8 reveals that velocity in the axial direction monotonically increases as slip velocity increases. This figure further indicates that for large values of slip velocity factor $\left(S_{f}\right)$, the frictional resistance between the viscous fluid and the surface is eliminated and shrinking of the sheet does no longer impose any motion of the fluid. It is also seen that for no slip condition i,e, for $S_{f}=0.0$, flow separation is observed adjacent to the lower wall. We have a similar observation in respect of the variation of the induced magnetic field with the increase in slip factor (cf. Fig. 9). 


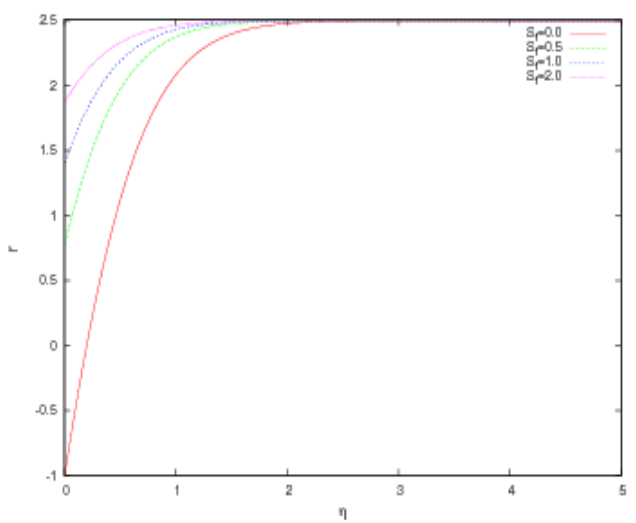

Fig. 8. Nature of axial velocity distribution for different values of $S_{f}$

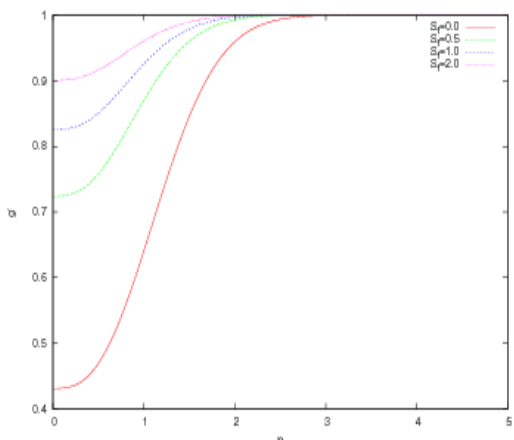

Fig. 9. Variation of induced magnetic field in $x$ -direction for different values of $S_{f}$

Figures. 10 to 12 give some characteristic temperature profiles for different values of Prandtl number ( $\mathrm{Pr}$ ), radiation parameter ( $\mathrm{Nr}$ ) and thermal slip factor $S_{t}$ respectively. Fig. 10 demonstrates the effect of Prandtl number ( $P r$ ) on temperature profile in the boundary layer. It is seen that the effect of Prandtl number is to decrease the temperature profile in The boundary layer. This can be attributed to the fact that the thermal boundary thickness decreases with increase in Prandtl number. Fig. 11 shows the effect of thermal radiation on temperature $\theta$. It is observed that the increase in thermal radiation parameter ( $\mathrm{Nr}$ ) produces a significant increase in the thickness of the thermal boundary layer of the fluid and so the temperature $\theta$ increases. Fig. 12 gives the temperature distribution for different values of thermal slip factor $\left(S_{t}\right)$. This figure shows that temperature decreases as the thermal slip increases.

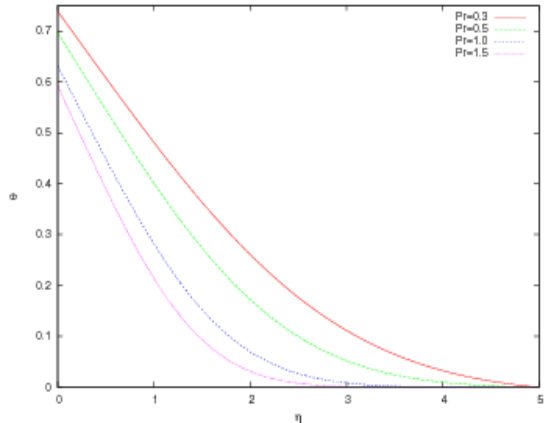

Fig. 10. Temperature distribution for different

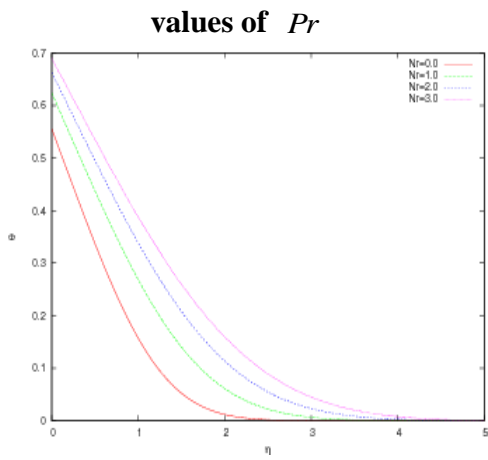

Fig. 11. Temperature distribution for different values of $\mathrm{Nr}$

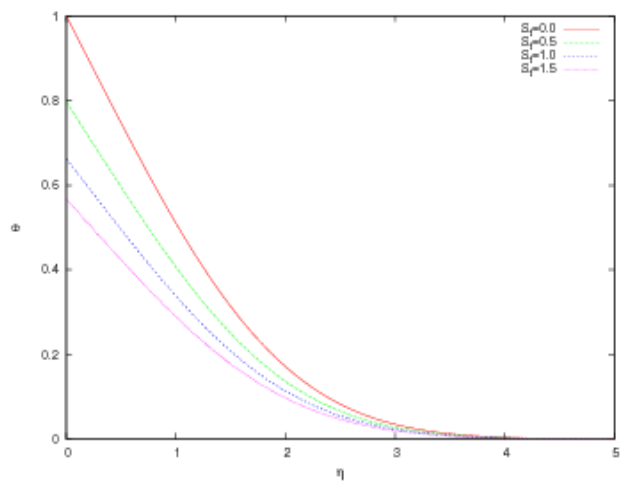

Fig. 12. Temperature distribution for different values of $S_{t}$

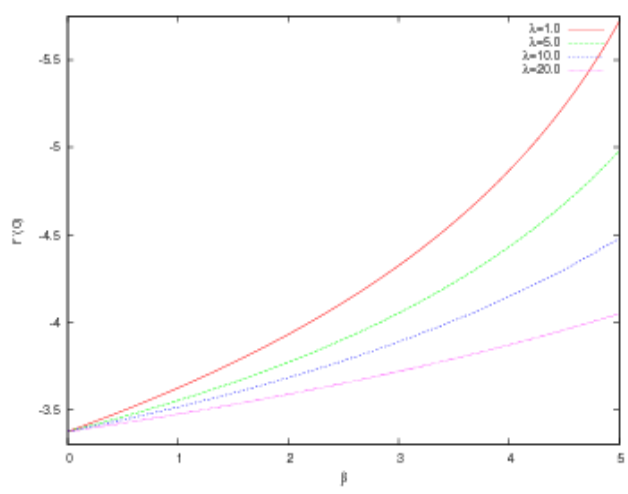

Fig. 13. Variation of skin-friction with $\beta$ for different values of $\lambda$

The skin-friction coefficient, defined as:

$C_{f}=\frac{\tau_{w}}{\rho U_{w}^{2} / 2}=\frac{1}{2} \operatorname{Re}_{x}^{-\frac{1}{2}} f^{\prime \prime}(0)$

where $\tau_{w}=\mu\left(\frac{\partial u}{\partial y}\right)_{y=0}$

is an important physical quantity that bears the potential to explore some vital information regarding problems such as the one under our present consideration.

Figure. 13 gives the variation of skin-friction with magnetic parameter $\beta$ for different values of the reciprocal magnetic Prandtl number $\lambda$. It is seen that skin-friction increases as $\lambda$ increases. It is also 
seen that skin-friction decreases as $\beta$ increases. Another important characteristic of the present study is the local Nusselt number $N u_{x}$, defined as

$N u_{x}=\frac{x q_{w}}{k\left(T_{w}-T_{\infty}\right)}=-\operatorname{Re}_{x}^{\frac{1}{2}} \theta^{\prime}(0)$

where $q_{w}=-k\left(\frac{\partial T}{\partial y}\right) y=0$

The values of $\theta^{\prime}(0)$ on the stretching wall, computed on the basis of the present study are presented in tabular form.

Table 1 Values of $-\boldsymbol{\theta}^{\prime} \frown 0$ ' for different values of $\beta, a / c, \operatorname{Pr}, \mathrm{Nr}$ when $\lambda=1.0, S_{f}=0.25$ and $S_{\mathrm{t}}=1.0$

\begin{tabular}{|l|l|l|l|l|}
\hline$\beta$ & $a / c$ & $P r$ & $\mathrm{Nr}$ & $-\theta(0)$ \\
\hline 2.0 & 2.5 & 0.7 & 2.0 & 0.32513349 \\
\hline 2.0 & 2.5 & 0.7 & 4.0 & 0.28118201 \\
\hline 2.0 & 2.5 & 1.0 & 2.0 & 0.35795494 \\
\hline 2.0 & 3.5 & 0.7 & 2.0 & 0.37871776 \\
\hline 2.5 & 2.5 & 0.7 & 2.0 & 0.32070163 \\
\hline
\end{tabular}

From Table 1, one can have an idea of the variation in local Nusselt number for different values of magnetic parameter $(\beta), a / c$, radiation parameter $(\mathrm{Nr})$ and Prandtl number $(\mathrm{Pr})$. This table shows that increase in $a / c$ or $\mathrm{Pr}$, enhances the local Nusselt number, while increase in magnetic parameter or radiation parameter leads to a reduction in local Nusselt number.

With an aim to validate our numerical model, we have compared our results for the axial velocity distribution with those reported recently by Nadeem and Hussain (2009) who carried out a similar study under some simplifying assumptions and obtained analytical solution by using the homotopy analysis method (HAM). We notice that the results of our numerical model are in excellent agreement with those reported by Nadeem and Hussain (2009) (cf. Fig. 14).

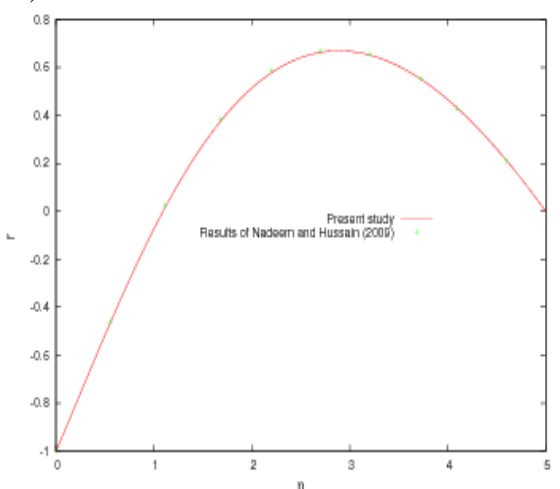

Fig. 14. Axial velocity distribution in the absence of induced magnetic field and velocity slip (when $a / c=0)$. (Comparison of the results of the present study with the analytical solution of Nadeem and Hussain (2009))

\section{SUMMARY AND CONCLUSION}

We have obtained an exact similarity solution of a steady two-dimensional magnetohydrodynamic stagnation-point flow of an incompressible electrically conducting fluid viscous fluid over a shrinking sheet in the presence of an induced magnetic field. The numerical analysis of a problem that deals with the investigation of the influence of velocity slip, thermal slip and thermal radiation on the flow field. The following conclusions can be drawn as a result of the numerical computations:

(1) In the presence of a magnetic field, the fluid velocity decreases. This is accompanied by a reduction in the velocity gradient at the wall, and thus the local skin-friction coefficient decreases.

(2) As Prandtl number increases, the local Nusselt number increases and thus the temperature gradient at the surface reduces. This implies that the ambient fluid gains temperature from the stretching sheet.

(3) As thermal radiation increases, thermal boundary layer thickness increase.

\section{ACKNOWLEDGEMENTS}

The author is grateful to the NBHM, DAE, Mumbai for the financial support of this investigation.

\section{REFERENCES}

Afify, A. A. (2004). MHD free-convective flow and mass transfer over a stretching sheet with chemical reaction. Heat Mass Transf. 40, 495-500.

Andersson, H. I., K. H. Bech and B. S. Dandapat (1992). Magnetohydrodynamic flow of a Powerlaw fluid over a stretching surface. Int. J. Non-Linear Mech. 27, 929-936.

Cobble, M. H. (1979). Free convection with mass transfer under the influence of a magnetic field. Nonlinear Analysis: Theory, Methods and Applications 3, 135-143.

Crane, L. J. (1970). Flow past a Stretching Plate. Zeitschrift fur Angewandte Mathematik und Physik (ZAMP) 21, 645-647.

Datti, P. S., K. V. Prasad, M. S. Abel and A. Joshi (2004). MHD visco-elastic fluid flow over a non-isothermal stretching sheet. Int. J. Eng. Sci. 42, 935-946.

Erfani, E., M. M. Rashidi and A. Basiri parsa (2010). The Modified Differential Transform Method for Solving Off-Centered Stagnation Flow towards a Rotating Disc. International Journal of Computational Methods 7 655-670.

Fang, T. and J. Zhang (2009). Closed-form exact solutions of MHD viscous flow over a shrinking sheet. Communications in Nonlinear Science and Numerical Simulation 14, 2853-2857. 
Hartmann, J. (1937). Hg-dynamics I, theory of the laminar flow of an electrically conducting liquid in a homogenous magnetic field. Matematisk-Fysiske Meddelelser 15 1-28.

Hiemenz, K. (1911). Die grenzschicht an einem in den gleichformigen flussigkeitsstrom eingetauchten geraden kreiszylinder. Polytechnic Journal 326, 321-324.

Ishak, A., R. Nazar and I. Pop (2008). Magnetohydrodynamic (MHD) Flow of a Micropolar Fluid Towards a Stagnation Point on a Vertical Surface. Comput. Math. Appl. $56,3188-3194$

Karwe, M. V. and Y. Jaluria (1988). Fluid flow and mixed convection transport from a moving plate in rolling and extrusion process. $A S M E$ Journal of Heat Transfer 110, 655-661.

Karwe, M. V. and Y. Jaluria (1988). Numerical simulation of thermal transport associated with a continuously moving flat sheet in materials processing. ASME Journal of Heat Transfer 113, 612-619.

Kumari, M. and G. Nath (2009). Steady mixed convection stagnation-point flow of upper convected Maxwell fluids with magnetic field. International Journal of Non-Linear Mechanics 44, 1048-1055.

Lin, E N. and B. T. Chao (1974). Laminar free convection over two-dimensional and axisymmetric bodies of arbitrary contour. Trans. ASME. Ser. C: J. Heat Transfer 94, 435-442.

Mahapatra, T. R. and A. S. Gupta (2004). Stagnation-point flow of a viscoelastic fluid towards a stretching surface. International Journal of Non-Linear Mechanics, 39, 811-820.

Miklavcic, M. and C. Y. Wang (2006). Viscous flow due to a shrinking sheet. Quarterly of Applied Mathematics 64, 283-290.
Nadeem, S. and A. Hussain (2009). MHD flow of a viscous fluid on a nonlinear porous shrinking sheet with homotopy analysis method. Appl. Math. Mech. -Engl. Ed. 30, 1569-1578.

Rajagopal, K. R., A. S. Gupta and A. S. Wineman (1980). On a boundary layer theory for non-Newtonian fluids. Appl.Sci.Eng.Lett. 18,875 .

Rashidi, M. M. and E. Erfani (2011). A New Analytical Study of MHD StagnationPoint Flow in Porous Media with Heat Transfer. Computers \& Fluids 40 172-178.

Rashidi, M. M., T. Hayat, E. Erfani, S. A. Mohimanian Pour, A. A-Hendi (2011). Simultaneous effects of partial slip and thermal-diffusion and diffusion-thermo on steady MHD convective flow due to a rotating disk. Communications in Nonlinear Science and Numerical Simulations 16 4303-4317.

Sajid, M., T. Hayat and T. Javed (2008). MHD rotating flow of a viscous fluid over a shrinking surface. Nonlinear Dynamics 51, 259-265.

Schlichting, H. and C. Gersten (2000). Boundary Layer Theory, eighthed. Springer,Berlin.

Sharma, P. R. and G. Singh (2009). Effects of Variable Thermal Conductivity and Heat Source/Sink on MHD Flow Near a Stagnation Point on a Linearly Stretching Sheet. Journal of Applied Fluid Mechanics 2, 13-21.

Singh, P., D. Singh and N. S. Tomer (2012). Oblique Stagnation-Point Darcy Flow towards a Stretching Sheet. Journal of Applied Fluid Mechanics 5, 29-37.

Zhu, J., L. C. Zheng and X. X. Zhang (2009). Analytical solution to stagnation-point flow and heat transfer over a stretching sheet based on homotopy analysis. Applied Mathematics and Mechanics (English Edition) 30, 463-474. 